\title{
Changement De Couverture Forestière Dans Le Département De La Likouala (République Du Congo) Durant La Période De 1986 À 2015
}

\author{
Leslie Bouetou-Kadilamio, PhD student \\ Laboratoire de Biologie et Ecologie Végétale, Université Marien \\ N'GOUABI, Faculté des Sciences, Brazzaville, République of the Congo \\ Suspense Averti Ifo, PhD \\ Ecole Normale Supérieure, Laboratoire de Géomatique et d'Ecologie \\ Tropicale Appliquée, \\ Université Marien N'GOUABI, Brazzaville, République of the Congo \\ Stoffenne Binsangou; PhD student \\ Ecole Normale Supérieure, Laboratoire de Géomatique et d'Ecologie \\ Tropicale Appliquée, Université Marien N'GOUABI
}

doi: 10.19044/esj.2017.v13n24p322 URL:http://dx.doi.org/10.19044/esj.2017.v13n24p322

\begin{abstract}
A study on the change of forest cover was carried out in the Department of Likouala, Republic of Congo for the period from 1986 to 2015. It was intended to calculate the areas of the different land conversions in the study period, but also to identify the causes of deforestation and forest land Landsat TM (1986), Etm + (2001), and Oli (2015) images were treated with the method of supervised classification "Maximum Likelihood" was applied to obtain the different maps of land type. The results revealed the lost of forest, with 133.624 hectares of intact forests in1986, 126980.89 ha of intact forests in 2001 and 114.166 ha of intact forests in 2015. There were also increases and regressions in areas of other types of land tenure.

This study shows that agriculture is the main driving force for the conversion of forest land to non-forest land. Given the importance of the coverage of lost forests, it appears urgent to strengthen the sustainable management of these ecosystems with a view to combating climate change.
\end{abstract}

Keywords: Forest, deforestation, Landsat, Likouala, Republic of Congo

\section{Résumé}

Une étude sur le changement de la couverture forestière a été réalisée dans le département de la Likouala en République du Congo dans la période de 1986 à 2015. Celle-ci avait pour but de calculer les superficies des 
différentes conversions des terres dans la période d'étude, mais aussi d'identifier les causes de la déforestation et de la dégradation des terres forestières. Les images Landsat TM (1986), ETM+ (2001) et OLI (2015) ont été traitées avec la méthode de classification supervisée "Maximum Vraisemblance" pour obtenir les différentes cartes d'occupation du sol. Les résultats révèlent que la forêt est passée de 133624 hectares de forêts intactes en 1986 à 126980,89 ha de forêt primaire en 2001 et à 114,166 ha de forêt en 2015. Par ailleurs on a noté des augmentations et des régressions de superficies des autres types d'occupation du sol. Il ressort de cette étude que l'agriculture est le principal moteur de la conversion des terres forestières vers les terres non forestières. $\mathrm{Vu}$ l'importance de la couverture des forêts perdues, il apparait urgent de renforcer la gestion durable de ces écosystèmes forestiers en vue d'une lutte contre les changements climatiques.

Mots clés: Forêt, Déforestation, Landsat, Likouala, République du Congo

\section{Introduction}

La situation du couvert forestier en République du Congo n'est pas uniforme sur l'ensemble du pays et varie en fonction de la densité de la population, de la qualité des infrastructures de base, du transport, de la richesse des forêts, de l'historique de leur exploitation enfin de l'existence de zones urbaines (NERF, 2016).

En effet, ces forêts subissent plusieurs changements et conversion des terres. Ces conversions peuvent avoir des impacts importants non seulement sur les flux et stocks de carbone, mais aussi sur la biocénose ainsi que sur les services écosystémiques de la forêt. Elles entrainent des émissions et absorptions des GES (GIEC, 2006).

Plusieurs études sur les changements de couverture forestière ont été réalisées en République du Congo. Une étude plus récente publiée par le CNIAF (2015) sur les pertes dans la période de 2000-2014 confirme des pertes de 295957 ha soit une moyenne annuelle de 21139 ha, avec un taux de perte de $0,062 \%$. D'autre part, Makaya (2015) affirme que ces pertes de forêts touchent $76 \%$ les forêts primaires et $24 \%$ les forêts secondaires. Elle affirme aussi que les principales causes de cette perte sont: les infrastructures (29\%), l'exploitation forestière (21\%) et l'agriculture (20\%).

Les causes de la déforestation et de la dégradation ont été étudiées par plusieurs auteurs, elles sont multiples et l'on distingue les causes directes et les causes indirectes. (Geist et Lambin, 2001; Zhang et Guindon, 2005 ; Duveiller et al., 2008 ; Tchatchou et $a l ., 2015$ ).

La présente étude qui a le mérite de s'étaler sur 29 ans a été menée dans le département de la Likouala qui a la plus vaste étendue du couvert forestier ( $85 \%$ du département). Cette étude a pour but : (i) de comprendre 
les moteurs de la déforestation dans cette région ainsi que leurs évolutions durant la période 1986-2015 dans l'axe Impfondo-Dongou, (ii) de déterminer les superficies des différentes conversions de terre.

\section{Matériels et méthodes}

\section{Zone d'étude}

La zone d'étude est située au nord de la République du Congo dans le Département de La Likouala qui couvre une superficie totale de 155274 ha. Elle est comprise entre $1^{\circ} 27^{\prime} 52,85^{\prime \prime}$ ' et $2^{\circ} 6{ }^{\prime} 55,76^{\prime}$ ' de latitude Nord et entre $17^{\circ} 52^{\prime} 35,04^{\prime}$ ' et $18^{\circ} 04^{\prime} 32,65^{\prime}$ 'de longitude Est. Cette zone regroupe les entités administratives d'Impfondo et de Dongou (figure 1).

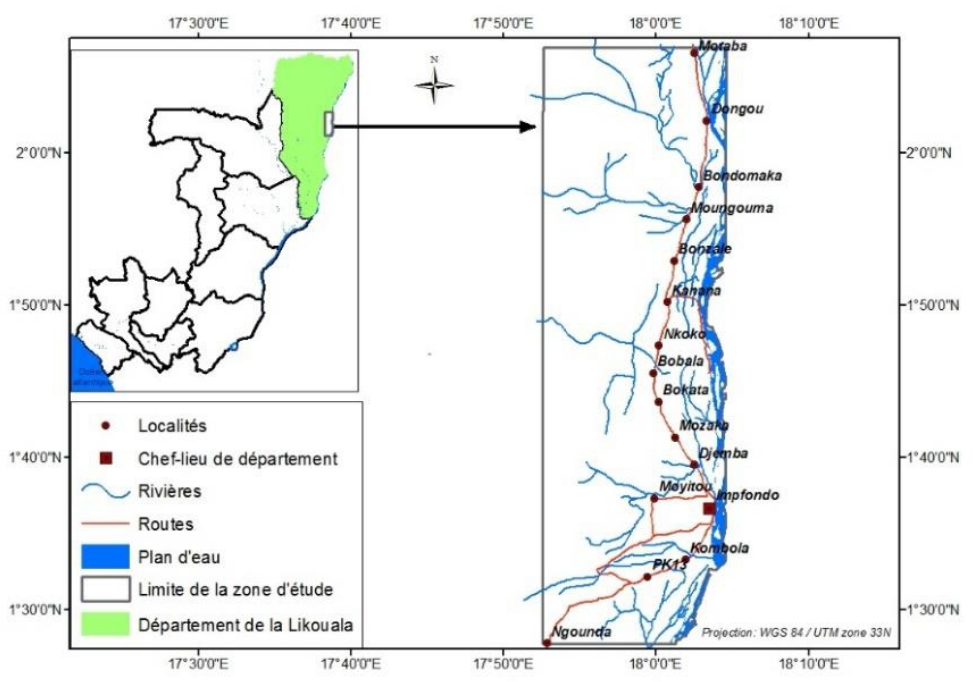

Figure 1: Localisation de la zone d'étude

\section{Climat}

Le climat qui règne dans le département s'apparente aux climats équatoriaux et tropicaux humides du type guinéen forestier (Figure 2).

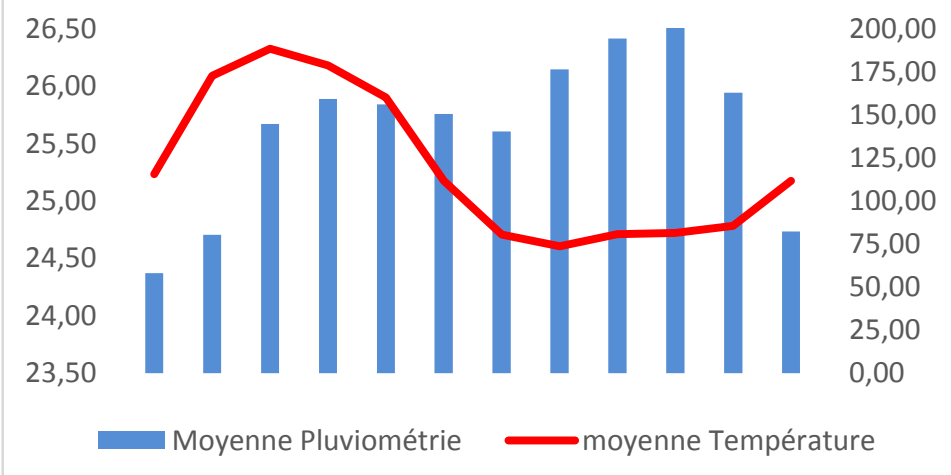

Figure 1 : Diagramme ombrothermique de la Likouala (moyenne de 1932-2015), (ANAC Congo 2016) 


\section{Végétation}

A l'exception de quelques rares espaces non forestiers, savanes, implantations humaines, cultures et jachères, lits des cours d'eau, sol nu (Nkokolo Nkaya, 2012), l'ensemble du Département de la Likouala est couverte par des forêts appartenant aux forêts denses humides sempervirentes (Vennetier, 1966). La végétation de la Likouala précisément vers Epena, présente un aspect très classique avec une succession en hauteur des diverses essences : l'étage dominant constitué des grands arbres de 40 à $60 \mathrm{~m}$ de hauteur tel que Limba (Teminalia superba), Iroko (Milicia excelsa), Ayous (Triplochiton scleroxylon), Azobé (Lophira alata), etc. La forêt marécageuse règne sur presque toute la Likouala (forêt inondée et forêt inondable) (Vennetier, 1966). La zone d'étude montre une variabilité d'écosystèmes forestiers partant de la forêt exondée à la forêt inondée et à la forêt dégradée pour les raisons agricoles.

\section{Données et outils utilisés}

Trois catégories de données ont servi à la réalisation de cette étude. La première catégorie est constituée des trois images satellitaires: une image TM du capteur Landsat datant du 18/12/1986, une image ETM+ datant du 18/02/2001 et une image OLI datant du 17/02/2015. Ces images satellitaires ont toutes été acquises en saison sèche. La seconde catégorie comprend des données dites auxiliaires. Elles sont composées des coordonnées géographiques (GPS), des observations directes sur le terrain et des cartes topographiques au 1/200.000 (feuilles d'Impfondo, de Dongou et de Gandou). La troisième catégorie a été obtenue lors la mission de terrain (septembre 2015) qui nous a permis de mieux discriminer les différents types d'occupation du sol de la zone d'étude. Les différentes données ont été traitées à l'aide des logiciels ENVI 5.1 et ArcGIS 10.2.

\section{Prétraitement des images}

Cette étape se résume en un ensemble d'opération, ayant pour but d'augmenter la lisibilité des images et de faciliter leur interprétation en vue d'une meilleure extraction de l'information. Les corrections radiométriques et atmosphériques ont été appliquées sur les images.

\section{Traitements des images satellitaires}

\section{Calculs des indices de végétation}

Deux indices ont été calculés, fournissant des informations sur les caractéristiques physiques et biologiques de la végétation: le Normalized Difference Vegetation Index ou indice de végétation normalisé NDVI $=(P I R$ $-\mathrm{R})$ / (PIR $+\mathrm{R})$, qui a pour effet d'augmenter le contraste au niveau du thème "végétation chlorophyllienne » et de réduire ceux des autres thèmes (Lacombre, 2008) et le Brightness Index ou indice de Brillance des sols IB = 
est: $\sqrt{R^{2}+P I R}$ applique l'effet contraire (Bannari et al., 1995) et met en évidence les éléments stériles (non chlorophylliens comme l'urbain et le sol $\mathrm{nu}$ ).

\section{Compositions colorées et la sélection des zones d'entraînement}

Les deux indices créés ont été associés à la bande du proche infrarouge dans une composition colorée RVB (IB, NDVI et PIR) afin de mieux identifier les grands types de végétation. Des zones d'entrainement homogènes ont été choisies sur la base des données GPS, des informations reçues auprès des populations et des photos prises sur le terrain. Lors de ces opérations de saisies des zones d'entraînement, une attention particulière a été portée aux changements dus aux différences temporelles surtout pour les images de 1986 et de 2001. Pour s'assurer d'un contenu équivalent des classes d'occupation du sol de 2015 par rapport à 1986 et 2001, seuls les groupes de pixels inchangés ont été prioritairement sélectionnés. La sélection de ces zones d'entrainement était faite autour du point GPS de façon à obtenir les pixels représentant la classe d'occupation du sol les plus larges possibles tout en restant suffisamment minutieux pour ne pas intégrer différents types de classe. Ainsi, 78 points GPS ont permis de saisir les zones d'entrainement homogènes dans la zone d'étude. C'est ainsi on distingue onze classes d'occupation du sol, dégagées en tenant compte de la classification de Yangambi (1956).

\section{Classification supervisées}

Sur la base des connaissances avérées du terrain, nous avons opté pour la classification supervisée avec l'algorithme du "maximum vraisemblance". Cet algorithme utilise les statistiques des échantillons de parcelles d'entrainement pour calculer la probabilité d'appartenance de chaque pixel à l'une des classes d'occupation du sol qui a la plus forte probabilité (Skupinski, 2009 ; Shalaby et al., 2007 ; Wu et al., 2006).

\section{Validation des classifications supervisées}

Pour valider les classifications supervisées, des comparaisons entre les zones d'entraînement de départ et les nouvelles zones de validation dans lesquelles sont vérifiées les statistiques des pixels bien classés. Les résultats obtenus sont contenus dans des matrices de confusion. Ces matrices ont permis de déterminer deux indices suivants : la précision globale (pourcentage de pixels correctement classés par rapport à ceux mal classés) et l'indice de Kappa (rapport entre le nombre de pixels bien classés et le total des pixels sondés) (Skupinski et al., 2009). L'indice de Kappa informe sur la concordance entre les données à classer et les données de référence (Congalton, 1991). 


\section{Post-traitement des images}

\section{Restitution cartographique des classifications}

Au cours de cette étape huit classes ont été finalement retenues : les forêts intactes ou primaires qui regroupent les forêts marécageuses et les forêts inondables, les forêts secondaires qui ne sont autres que les forêts dégradées, les cultures et jachères ; les plantations, les habitats, les savanes et l'eau. Trois cartes d'occupation du sol des années 1986, 2001 et 2015 sont ainsi produites.

\section{Evaluation de la dynamique de l'occupation du sol}

Les données obtenues de l'interprétation de chaque image Landsat TM (1986), ETM+ (2001) et OLI (2015) ont été analysées et comparées. Ainsi, pour chaque unité d'occupation du sol, le taux d'évolution annuel est calculé selon la formule de l'équation suivante:

$$
\mathrm{T}_{\text {annuel }}=\left(\mathrm{V}_{\mathrm{f}}-\mathrm{V}_{\mathrm{i}}\right) / \mathrm{P} * 100
$$

Où Vf représente la valeur de la statistique de la strate finale, Vi la valeur des statistiques de la strate initiale et $\mathrm{P}$ est la durée de la période d'observation entre les dates. Cette formule permet de faire une analyse diachronique de la dynamique d'occupation des sols sur la zone étudiée.

\section{Mise en évidence des changements d'occupation du sol}

Les croisements deux à deux des trois cartes d'occupation du sol (1986-2001 ; 2001-2015 ; 1986-2015) sous le logiciel ArcGIS nous ont permis d'élaborer des cartes de changements d'occupation du sol.

\section{Résultats}

\section{Evaluation de la précision de la classification}

Les matrices de confusion ont été élaborées pour évaluer la précision de la classification (Tableaux 1, 2 et 3). Il ressort de cette analyse que les trois images présentent une bonne précision globale pour l'ensemble de la zone d'étude. Ainsi, l'image TM (1986) a une précision globale de 88,02\% avec un indice de Kappa évalué à 86,60\%, alors que l'image ETM+ (2001) a une précision globale de $92,15 \%$ avec un indice de Kappa évalué à 91,11\% et l'image OLI (2015) a une précision globale de 93,56\% avec un indice de Kappa évalué à 92,64\%.

$\mathrm{Au}$ niveau des différentes classes la précision n'est pas identique. Certaines classes d'occupation du sol sont classifiées avec plus de précision que d'autres. De même, les classes de la matrice de confusion de l'image OLI sont mieux classifiées que celles de l'image ETM+ et TM. 
Une analyse plus fine de ces données montre que, la classe des forêts intactes (forêt marécageuse et forêt inondable) est mieux classifiée dans les trois dates c'est-à-dire plus de $90 \%$ de précision, alors que la classe forêt dégradée et la classe plantation présentent de fortes confusions. En effet, $34 \%$ des pixels de forêts dégradée se retrouvent classés dans les plantations, d'où le faible pourcentage de pixels de forêts bien classés (58\%) sur l'image de 1986 (tableau 1). La classe de culture-jachère présente également de faibles pourcentages de pixels bien classés (61\%) compte tenu de leur confusion avec la classe de forêt dégradée. Les autres types d'occupation du sol sont en général bien discriminés ou classifiés. Sur l'image de 2001, les plus faibles précisions ont été observées au niveau de classe savane où $12,18 \%$ et $10,55 \%$ des pixels de savane se retrouvent classés dans les classes cultures et jachères et habitat (tableau 2). Alors que, sur l'image de 2015, les plus faibles précisions ont été observées au niveau de la classe culture et jachère. On remarque en outre que la classe plantation présentait une confusion de $14 \%$ avec la classe forêt dégradée (tableau 3). Cette confusion peut être due à la présence de cultures de cacao et de palmier à huile qui se comporteraient comme des forêts dégradées (tableau 3).

Dans une étude de l'occupation des sols, lorsque l'indice de Kappa évalué dans les opérations de classification est compris entre 50 et $75 \%$, la classification adoptée est valable et les résultats peuvent être judicieusement utilisés (Pontus, 2000). Dans la présente étude, les indices de Kappa dépassent $85 \%$. Les différents résultats d'évaluation des images obtenues par la méthode de la classification supervisée sont donc validés.

Tableau 1 : Matrice de confusion de la classification de l'image de 1986

\begin{tabular}{|c|c|c|c|c|c|c|c|c|c|c|c|}
\hline Classes & $\mathbf{1}$ & $\mathbf{2}$ & $\mathbf{3}$ & $\mathbf{4}$ & $\mathbf{5}$ & $\mathbf{6}$ & $\mathbf{7}$ & $\mathbf{8}$ & $\mathbf{9}$ & $\mathbf{1 0}$ & Total \\
\hline FP & $\mathbf{1 0 0}$ & 2,81 & 0,59 & 0 & 0 & 0 & 0 & 0 & 0,33 & 4,22 & 13,3 \\
\hline FD & 0 & $\mathbf{5 8 , 9}$ & 31,1 & 0 & 0 & 0 & 0 & 0 & 0 & 0 & 10,8 \\
\hline Ch & 0 & 4,16 & $\mathbf{6 1 , 8}$ & 0 & 0 & 0,14 & 9,75 & 0 & 0,16 & 0 & 8,83 \\
\hline PI & 0 & 34,1 & 1,3 & $\mathbf{1 0 0}$ & 0 & 0 & 0 & 0 & 0 & 0 & 6,05 \\
\hline Sa & 0 & 0 & 1,65 & 0 & $\mathbf{1 0 0}$ & 0 & 0 & 0,12 & 0 & 0 & 12,6 \\
\hline Ha & 0 & 0 & 3,18 & 0 & 0 & $\mathbf{9 1 , 8}$ & 2,87 & 0 & 1,15 & 0 & 10,2 \\
\hline BS & 0 & 0 & 0,35 & 0 & 0 & 8,08 & $\mathbf{8 7 , 2}$ & 0 & 0,16 & 0 & 7,48 \\
\hline Eau & 0 & 0 & 0 & 0 & 0 & 0 & 0,19 & $\mathbf{9 9 , 9}$ & 0 & 0 & 12,3 \\
\hline Nu & 0 & 0 & 0 & 0 & 0 & 0 & 0 & 0 & $\mathbf{9 7 , 9}$ & 0 & 8,57 \\
\hline ON & 0 & 0 & 0 & 0 & 0 & 0 & 0 & 0 & 0,33 & $\mathbf{9 5 , 8}$ & 9,86 \\
\hline Total & 100 & 100 & 100 & 100 & 100 & 100 & 100 & 100 & 100 & 100 & $\mathbf{1 0 0}$ \\
\hline
\end{tabular}

Précision globale : $88,02 \%$; Coefficient de Kappa : 0,866 
Tableau 2: Matrice de confusion de la classification de l'image de 2001

\begin{tabular}{|c|c|c|c|c|c|c|c|c|c|c|c|}
\hline Classes & $\mathbf{1}$ & $\mathbf{2}$ & $\mathbf{3}$ & $\mathbf{4}$ & $\mathbf{5}$ & $\mathbf{6}$ & $\mathbf{7}$ & $\mathbf{8}$ & $\mathbf{9}$ & $\mathbf{1 0}$ & Total \\
\hline FP & $\mathbf{9 3 , 5 2}$ & 6,33 & 0,76 & 0,45 & 0 & 0 & 0 & 0 & 0 & 0,61 & 9,53 \\
\hline FD & 6,48 & $\mathbf{8 8 , 0 3}$ & 11,33 & 9,55 & 0 & 0 & 0 & 0 & 0 & 0 & 11,12 \\
\hline Ch & 0 & 2,75 & $\mathbf{8 7 , 6 1}$ & 1,36 & 12,18 & 0 & 0 & 0,59 & 3,11 & 0 & 10,12 \\
\hline Pl & 0 & 2,89 & 0 & $\mathbf{8 8 , 6 4}$ & 0 & 0 & 0 & 0 & 0 & 0 & 3,09 \\
\hline Sa & 0 & 0 & 0,3 & 0 & $\mathbf{7 7 , 2 7}$ & 0 & 0 & 10,43 & 0 & 0 & 8,47 \\
\hline Ha & 0 & 0 & 0 & 0 & 10,55 & $\mathbf{1 0 0}$ & 5,41 & 1,17 & 0 & 0 & 10,93 \\
\hline BS & 0 & 0 & 0 & 0 & 0 & 0 & $\mathbf{9 2 , 4 3}$ & 0 & 0 & 0 & 8,55 \\
\hline Eau & 0 & 0 & 0 & 0 & 0 & 0 & 0 & $\mathbf{8 7 , 8 1}$ & 0 & 0 & 8,55 \\
\hline Nu & 0 & 0 & 0 & 0 & 0 & 0 & 2,16 & 0 & $\mathbf{9 6 , 8 9}$ & 0 & 8,66 \\
\hline ON & 0 & 0 & 0 & 0 & 0 & 0 & 0 & 0 & 0 & $\mathbf{9 9 , 3 9}$ & 20,99 \\
\hline Total & 100 & 100 & 100 & 100 & 100 & 100 & 100 & 100 & 100 & 100 & $\mathbf{1 0 0}$ \\
\hline
\end{tabular}

Précision globale : 92,15\% ; Coefficient de Kappa : 0,911

Tableau 3 : Matrice de confusion de la classification de l'image de 2015

\begin{tabular}{|c|c|c|c|c|c|c|c|c|c|}
\hline Classes & $\mathbf{1}$ & $\mathbf{2}$ & $\mathbf{3}$ & $\mathbf{4}$ & $\mathbf{5}$ & $\mathbf{6}$ & $\mathbf{7}$ & $\mathbf{8}$ & Total \\
\hline FP & $\mathbf{9 9}$ & 3,24 & 0 & 0,3 & 0 & 0 & 0 & 0 & 12,7 \\
\hline FD & 1,2 & $\mathbf{8 6}$ & 16,9 & 14 & 0 & 0 & 0 & 0 & 14,3 \\
\hline Ch & 0 & 3,04 & $\mathbf{8 2 , 3}$ & 1,9 & 0,9 & 0,09 & 0 & 0 & 11 \\
\hline PI & 0 & 7,75 & 0,19 & $\mathbf{8 4}$ & 0 & 0 & 0 & 0 & 10,3 \\
\hline Sa & 0 & 0 & 0,38 & 0 & $\mathbf{9 9}$ & 0 & 1,04 & 0,35 & 12,6 \\
\hline Ha & 0 & 0 & 0,29 & 0 & 0 & $\mathbf{9 9 , 9}$ & 0 & 1,74 & 13,3 \\
\hline BS & 0 & 0 & 0 & 0 & 0 & 0 & $\mathbf{9 9}$ & 0 & 12,5 \\
\hline Eau & 0 & 0 & 0 & 0 & 0 & 0 & 0 & $\mathbf{9 7 , 9}$ & 13,5 \\
\hline Total & 100 & 100 & 100 & 100 & 100 & 100 & 100 & 100 & $\mathbf{1 0 0}$ \\
\hline
\end{tabular}

Précision globale : 93,56\% ; Coefficient de Kappa : 0,9264

Avec :

$\mathrm{FP}=$ Forêt primaire (forêt marécageuse et forêt inondable) $; \mathrm{FD}=$ Forêt dégradée $; \mathrm{Ch}=$ champ ; $\mathrm{Pl}=$ plantation $; \mathrm{Sa}=$ savane $; \mathrm{Ha}=$ habitat $; \mathrm{BS}=$ bande sable; $\mathrm{Nu}=$ nuage $; \mathrm{ON}=$ ombre de nuage.

\section{Dynamique de l'occupation du sol dans le département de la Likouala Cartographie de l'occupation du sol en 1986}

En 1986, la zone d'étude est forestière à 85,99\% avec 133,624 hectares de forêts intactes qui représentent le type d'occupation du sol le plus important (figure 3). Ces forêts intactes (primaires) regroupent les forêts marécageuses et les forêts inondables dont leur physionomie et leurs caractéristiques floristiques sont très différentes des forêts de terre ferme. 
Les cultures-jachères sont moins importantes avec 3,421 ha. Les savanes représentent une superficie moins importante avec 3,947 ha de la superficie totale de la zone. Les forêts secondaires sont constituées de 5,700 ha de la superficie totale de la zone. Il s'agit d'un faciès dégradé des forêts dense sempervirentes et semi- sempervirentes.

A cette date les plantations, l'habitat et les bandes de sable représentent des infimes parties et ont été estimé respectivement à 893 ha, 852 ha et 624 ha de la superficie totale de la zone d'étude.

\section{Cartographie de l'occupation du sol en 2001}

En 2001, la zone est toujours dominée par la forêt intacte $81,71 \%$ avec 126980,89 ha de la superficie totale de la zone d'étude (figure 4). Les superficies des cultures-jachères $(7556,81 \mathrm{ha})$ et forêts dégradées $(8357,92$ ha) sont importantes par rapport à celles de 1986. Les superficies de plantation, d'habitat et de bandes de sable représentent des infimes parties et ont été estimées respectivement à 269,35ha, 1064,62 ha, 1976,49 ha mais sont plus importantes que celles de 1986.
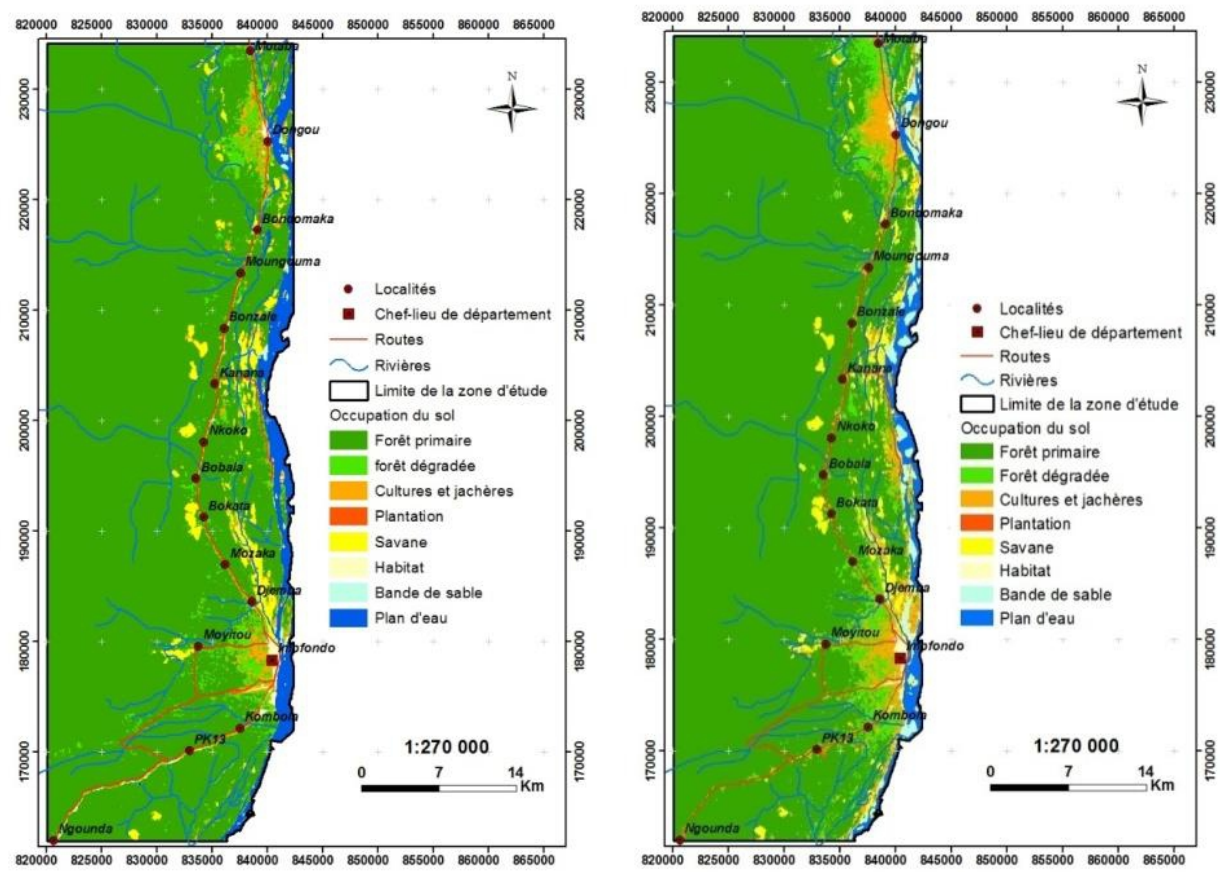

Figure 3: Carte d'occupation du sol en 1986 Figure 4: Carte d'occupation du sol en 2001

\section{Cartographie de l'occupation du sol en 2015}

La cartographie de l'occupation du sol en 2015 montre que les milieux naturels constitués de forêts intactes et de savane sont plus ou moins bien conservés, couvrant 114.166 ha soit $73,47 \%$ de la superficie totale de la zone pour les forêts intactes et 3.622 ha soit $2,33 \%$ de la superficie totale de 
la zone pour les savanes (figure 5). Physionomiquement, ces forêts observées constituent deux ensembles majeures : les forêts marécageuses et les forêts inondables évoluant toutes vers les faciès secondaires (dégradés). Les savanes observées sont les savanes herbeuses incluses. L'habitat s'étend sur $1,25 \%$ de la superficie totale de la zone. La majorité de terres autour du département (Impfondo), des localités et le long de la route est occupée par l'agriculture avec $7,82 \%$ des terres de la superficie totale de la zone d'étude. Cette agriculture est pratiquée pour la plupart sur les surfaces modestes par des paysans utilisant des outils traditionnels et pratiquant l'agriculture sur brulis. Les cultures pratiquées sont surtout le manioc, le maïs, le cacao, la banane, le taro, le palmier à huile, les arbres fruitiers (safou, oranger, etc).

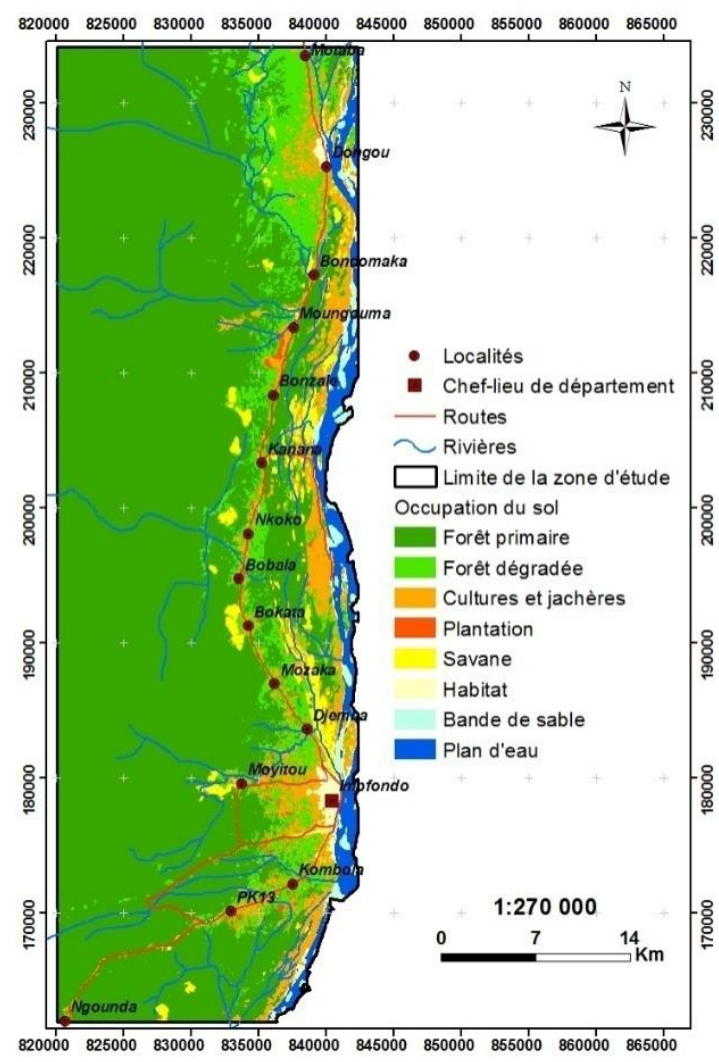

Figure 5: Carte d'occupation du sol en 20150.

\section{Evaluation de la dynamique de l'occupation du sol de 1986-2001-2015}

L'examen des cartes d'occupation du sol (Figures 3 et 4) et le Tableaux 4 associé à cette figure 6 montre une modification de l'occupation du sol en général et du couvert végétal entre 1986 et 2001. Ainsi, on constate une réduction de la forêt intacte ou la forêt primaire et l'eau en quinze ans. La forêt est passée de $85,98 \%$ en 1986 à $81,71 \%$ en 2001 et l'eau de $4,08 \%$ en 1986 à 2,03\% en 2001. On observe aussi une augmentation des 
superficies occupées par les forêts dégradées, les cultures-jachères, les habitats, les plantations et les bandes de sable qui passent de 3,91\% à 5,38\%, $2,26 \%$ à $4,86 \%, 0,55 \%$ à $0,69 \%, 0,28 \%$ à $0,17 \%$ et $0,40 \%$ à $1,27 \%$ respectivement.

Le tableau 4 indique des diminutions annuelles de 0,28\%, 0,01\%, et $0,07 \%$ respectivement pour la classe forêt, plantation et eau. On note une augmentation moyenne annuelle de $0,10 \%, 0,17 \%, 0,03 \%, 0,01 \%$ et $0,06 \%$ respectivement pour la classe forêt dégradée, cultures-jachère, savane, habitat et bande de sable.

L'analyse des cartes d'occupation du sol (Figures 4 et 5) et le Tableaux 4 associé à cette figure 6 montrent une modification du couvert végétal entre 2001 et 2015. Ainsi, on constate une réduction de forêts intactes, de savanes et de Bande de sable en quatorze ans. La forêt est passée de $81,71 \%$ en 2001 à $73,47 \%$ en 2015 , la savane de $2,92 \%$ en 2001 à $2,33 \%$ en 2015 et les bandes de sable de $1,27 \%$ en 2001 à $1,02 \%$ en 2015 . On observe aussi une augmentation des superficies occupées par les forêts dégradées, les cultures et jachères, les habitats, les plantations et l'eau qui passent de $5,38 \%$ à $10,13 \%, 4,86 \%$ à $7,82 \%, 0,69 \%$ à $1,25 \%, 0,17 \%$ à $0,60 \%$ et $2,99 \%$ à $3,39 \%$ respectivement.

Le tableau 4 indique des réductions annuelles de $0,59 \%, 0,04 \%$, et $0,02 \%$ respectivement pour la classe forêt, savanes et bande de sable. On note une augmentation moyenne annuelle de $0,34 \%, 0,21 \%, 0,03 \%, 0,04 \%$ et $0,03 \%$ respectivement pour la classe forêt dégradée, cultures-jachère, plantation, habitat et eau.

L'analyse des cartes d'occupation du sol (Figures 3 et 5) et le Tableaux 4 associé à cette figure 6 qui montre une modification du couvert végétal entre 1986 et 2015. Ainsi, on constate une régression de forêts primaires, de savanes et de l'eau en vingt-neuf ans. La forêt est passée de $85,98 \%$ en 1986 à $73,47 \%$ en 2015 , la savane de $2,54 \%$ en 1986 à $2,33 \%$ en 2015 et l'eau de 4,08\% en 1986 à 3,39\% en 2015. On observe aussi une augmentation des superficies occupées par les forêts dégradées, les culturesjachères, les habitats, les plantations et les bandes de sable qui passent de $3,91 \%$ à $10,13 \%, 2,26 \%$ à $7,82 \%, 0,55 \%$ à $1,25 \%, 0,17 \%$ à $0,60 \%$ et $0,40 \%$ à $1,02 \%$ respectivement.

Le tableau 4 indique des diminutions annuelles de 0,43\%, 0,01\%, et $0,02 \%$ respectivement pour la classe forêt, savanes et eau. On note une augmentation moyenne annuelle de $0,21 \%, 0,19 \%, 0,01 \%, 0,02 \%$ et $0,02 \%$ respectivement pour la classe forêt dégradée, cultures-jachère, plantation, habitat et bande de sable. 
Tableau 4: Taux d'évolution des classes de l'occupation du sol entre 1986-2001, 2001-2015 et $1986-2015$

\begin{tabular}{|c|c|c|c|c|c|c|}
\hline \multirow{2}{*}{ Classes } & \multicolumn{2}{|c|}{ Année 1896-2001 } & \multicolumn{2}{c|}{ Année 2001-2015 } & \multicolumn{2}{c|}{ Année 1986-2015 } \\
\cline { 2 - 7 } & $\mathrm{Tb}(\%)$ & $\mathrm{Ta}(\%)$ & $\mathrm{Tb}(\%)$ & $\mathrm{Ta}(\%)$ & $\mathrm{Tb}(\%)$ & $\mathrm{Ta}(\%)$ \\
\hline $\mathrm{FP}$ & $-4,27$ & $-0,28$ & $-8,25$ & $-0,59$ & $-12,51$ & $-0,43$ \\
\hline $\mathrm{FD}$ & 1,47 & 0,10 & 4,75 & 0,34 & 6,23 & 0,21 \\
\hline $\mathrm{Ch}$ & 2,60 & 0,17 & 2,95 & 0,21 & 5,56 & 0,19 \\
\hline $\mathrm{Pl}$ & $-0,11$ & $-0,01$ & 0,43 & 0,03 & 0,32 & 0,01 \\
\hline $\mathrm{Sa}$ & 0,38 & 0,03 & $-0,59$ & $-0,04$ & $-0,21$ & $-0,01$ \\
\hline $\mathrm{Ha}$ & 0,14 & 0,01 & 0,56 & 0,04 & 0,70 & 0,02 \\
\hline $\mathrm{BS}$ & 0,87 & 0,06 & $-0,26$ & $-0,02$ & 0,61 & 0,02 \\
\hline $\mathrm{Eau}$ & $-1,09$ & $-0,07$ & 0,40 & 0,03 & $-0,69$ & $-0,02$ \\
\hline
\end{tabular}

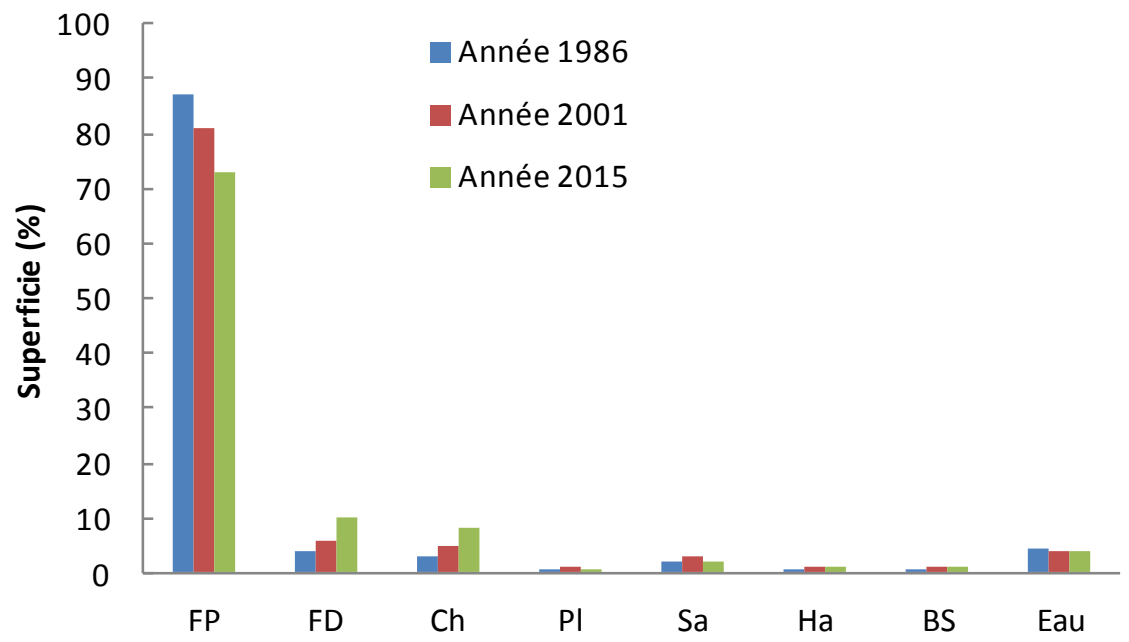

Classes d'occupations du sol

Figure 6 : Evolution des pourcentages des surfaces couvertes par chaque type d'occupation du sol de 1986-2001-2015

\section{Changements d'état des classes d'occupation du sol}

$\mathrm{Au}$ cours de la période 1986- 2001, les formations forestières primaires ont été les plus stables avec un taux de 79,13\% (tableau 5) tandis que la majeure partie des forêts primaires a été convertie en forêt dégradée $(1,20 \%)$, en culture-jachère $(0,52 \%)$ et en savane $(0,65 \%)$. Au niveau de la classe forêt dégradée, $0,41 \%$ a été convertie en culture-jachère et $0,11 \%$ de plantations a été convertie en culture-jachère entre 1986 et 2001. Cependant, la restauration de la végétation $(9,08 \%)$ s'est produite dans une moindre mesure grâce à la conversion de toutes les autres classes (forêts dégradées, de cultures et jachères, de savanes, de l'habitat, de bande de sable et de l'eau) en forêts primaires. 
La classe culture-jachère est convertie en plantations $(0,11 \%)$ en savane $(0,45 \%)$, et $0,41 \%$ en forêt dégradée. cela peut alors correspondre à des parcelles de culture abandonnées (longue période de jachère), une baisse de la pression sur cette classe entrainant sa reprise. Au niveau de la classe plantation $0,11 \%$ est convertie en culture-jachère et $0,02 \%$ est modifiée en forêt dégradée. Cela peut alors correspondre à des parcelles de culture de rente comme le palmier à huile et celles de cacao. Pour la classe savanes, $0,45 \%$ des surfaces savanicoles ont été converties en cultures-jachères qui sont soit des jachères jeunes et $0,88 \%$ a été modifiée en forêt primaire. La classe eau, 2,62\% de la surface initiale se trouve inchangée en 2001.

D'après l'analyse de cette matrice de transition, en quinze ans, plusieurs observations peuvent être faites :

Le maintien de la classe forêt primaire $(79,13 \%)$, tandis que près de $5,46 \%$ de cette classe a été convertie en d'autres classes (forêts dégradées, de cultures et jachères, de savanes, de l'habitat, de bande de sable et de l'eau).

Entre 2001 et 2015, 72,39\% des forêts primaires sont restées intactes, tandis que $0,89 \%$ sont devenues forêts dégradées et $0,21 \%$ ont été totalement converties en classe non forestière (tableau 6). Les forêts dégradées ont été modifiées en forêts primaires $(6,11 \%)$ et en cultures-jachères $(1 \%)$. Quant à la classe " cultures-jachères », sa proportion est passée de 4,88 à 7,78\% durant la même période. Enfin, la classe « plantations », avec 0,04\% de taux de permanence, a constitué la moins stable dans la zone d'étude. De manière globale cette période (2001-2015) se caractérise par trois principaux types de dynamiques forestières à savoir la stabilité des classes $(83,13 \%)$, la dynamique de densification de la couverture végétale $(9,32 \%)$ et la perturbation du milieu $(3,84 \%)$.

En examinant ce tableau 7, on observe quatre grands processus qui se sont déroulés dans la zone d'étude en 29 ans. Il s'agit : i-du maintien de la forêt primaire $(71,85 \%)$; ii- de la dégradation de l'écosystème forestier ou la perte du couvert forestier $(1,66 \%)$; iii- de la régénération naturelle ou anthropique du couvert forestier qui a abouti à la restauration et à la formation de forêt primaire $(14,13 \%)$; iiii- de l'ensablement et le dessèchement des plans d'eau et zones marécageuses qui ont entraîné une perte progressive des surfaces en eau.

Tableau 5 : Matrice de transition de l'occupation du sol (\%) pour les périodes, 1986-2001,

\begin{tabular}{|c|c|c|c|c|c|c|c|c|c|}
\hline $\begin{array}{l}2001 \\
1986\end{array}$ & 1 & 2 & 3 & 4 & 5 & 6 & 7 & 8 & Total \\
\hline FP & 79,13 & 1,20 & 0,52 & 0,03 & 0,65 & 0,09 & 0,05 & 0,05 & 81,71 \\
\hline FD & 3,51 & 1,22 & 0,41 & 0,09 & 0,09 & 0,04 & 0,02 & 0,01 & 5,38 \\
\hline Ch & 2,03 & 1,19 & 0,86 & 0,11 & 0,45 & 0,09 & 0,04 & 0,11 & 4,88 \\
\hline PI & 0,14 & 0,02 & 0,01 & 0,00 & 0,00 & 0,00 & 0,00 & 0,00 & 0,17 \\
\hline Sa & 0,88 & 0,15 & 0,26 & 0,01 & 1,26 & 0,04 & 0,04 & 0,27 & 2,91 \\
\hline
\end{tabular}




\begin{tabular}{|c|c|c|c|c|c|c|c|c|c|}
\hline Ha & 0,07 & 0,05 & 0,13 & 0,00 & 0,06 & 0,26 & 0,05 & 0,06 & 0,69 \\
\hline BS & 0,10 & 0,04 & 0,05 & 0,00 & 0,01 & 0,01 & 0,11 & 0,95 & 1,27 \\
\hline Eau & 0,14 & 0,04 & 0,04 & 0,00 & 0,02 & 0,02 & 0,10 & 2,62 & 2,99 \\
\hline Total & 85,98 & 3,92 & 2,27 & 0,25 & 2,54 & 0,55 & 0,40 & 4,08 & 100,00 \\
\hline
\end{tabular}

Tableau 6: Matrice de transition de l'occupation du sol (\%) pour les périodes, 2001-2015,

\begin{tabular}{|c|c|c|c|c|c|c|c|c|c|}
\hline 2001 & 1 & 2 & 3 & 4 & 5 & 6 & 7 & 8 & Total \\
\hline FP & 72,39 & 0,89 & 0,19 & 0,00 & 0,02 & 0,00 & 0,00 & 0,00 & 73,50 \\
\hline FD & 6,11 & 2,88 & 1,00 & 0,07 & 0,07 & 0,00 & 0,00 & 0,00 & 10,13 \\
\hline Ch & 2,77 & 1,42 & 2,77 & 0,06 & 0,66 & 0,08 & 0,02 & 0,01 & 7,78 \\
\hline PI & 0,33 & 0,14 & 0,08 & 0,04 & 0,01 & 0,00 & 0,00 & 0,00 & 0,60 \\
\hline Sa & 0,08 & 0,02 & 0,44 & 0,00 & 1,68 & 0,09 & 0,13 & 0,01 & 2,45 \\
\hline Ha & 0,03 & 0,04 & 0,39 & 0,00 & 0,17 & 0,47 & 0,01 & 0,03 & 1,13 \\
\hline BS & 0,00 & 0,00 & 0,00 & 0,00 & 0,06 & 0,01 & 0,45 & 0,49 & 1,02 \\
\hline Eau & 0,00 & 0,00 & 0,01 & 0,00 & 0,24 & 0,03 & 0,65 & 2,45 & 3,39 \\
\hline Total & 81,71 & 5,38 & 4,88 & 0,17 & 2,91 & 0,69 & 1,27 & 2,99 & 100,00 \\
\hline
\end{tabular}

Tableau 7 : Matrice de transition de l'occupation du sol (\%) pour les périodes, 1986-2015

\begin{tabular}{|c|c|c|c|c|c|c|c|c|c|}
\hline $\begin{array}{l}2015 \\
1986\end{array}$ & 1 & 2 & 3 & 4 & 5 & 6 & 7 & 8 & Total \\
\hline FP & 71,85 & 0,85 & 0,31 & 0,02 & 0,37 & 0,05 & 0,03 & 0,03 & 73,50 \\
\hline FD & 7,79 & 1,35 & 0,51 & 0,10 & 0,27 & 0,05 & 0,03 & 0,04 & 10,13 \\
\hline Ch & 4,75 & 1,29 & 0,83 & 0,10 & 0,53 & 0,10 & 0,06 & 0,12 & 7,78 \\
\hline PI & 0,53 & 0,05 & 0,01 & 0,00 & 0,00 & 0,00 & 0,00 & 0,00 & 0,60 \\
\hline Sa & 0,57 & 0,12 & 0,24 & 0,01 & 1,32 & 0,02 & 0,01 & 0,03 & 2,33 \\
\hline $\mathbf{H a}$ & 0,22 & 0,18 & 0,29 & 0,02 & 0,03 & 0,28 & 0,07 & 0,16 & 1,25 \\
\hline BS & 0,05 & 0,02 & 0,02 & 0,00 & 0,01 & 0,01 & 0,05 & 0,87 & 1,02 \\
\hline Eau & 0,22 & 0,06 & 0,06 & 0,00 & 0,02 & 0,03 & 0,16 & 2,83 & 3,39 \\
\hline Total & 85,98 & 3,92 & 2,27 & 0,25 & 2,54 & 0,55 & 0,40 & 4,08 & 100,00 \\
\hline
\end{tabular}

\section{Discussion}

\section{Approche méthodologique}

Les précisions globales des classifications sont respectivement de $88,02 \%$ pour $1986,92,15 \%$ pour 2001 et $93,56 \%$ pour 2015 . Ces précisions sont acceptables car selon Congalton (1991), une classification est jugée acceptable lorsque la précision globale avoisine $80 \%$. Selon les échelles de Pontus (2000), les résultats de cette analyse sont statistiquement acceptables. Ces précisions corroborent avec celles obtenues par d'autres auteurs tels que 
N'Guessan et al., (2006), N'DA (2007), Akadje et al., (2014) et Adjonou et al., (2010).

Il convient cependant de noter que les grandes précisions cartographiques obtenues peuvent aussi dépendre du nombre réduit de classes utilisées et de la définition de parcelles homogènes lors du choix des sites d'entraînement (Caloz et Collet, 2001).

L'analyse de la matrice de confusion aux différentes dates présente des pixels bien classés à plus de $70 \%$ par classe, avec une part plus importante de classe ayant des précisions supérieures à $90 \%$. En dépit du fait que ces résultats soient acceptables, il existe une grande confusion entre les classes forêt dégradée et culture-jachère et entre les classes forêt dégradée et plantation. En effet $31,1 \%$ et $34,11 \%$ de pixels de thème culture-jachère et plantation en 1986 ont été commis à la classe forêt dégradée (tableau 1). Ces différentes classes sont des formations végétales boisées, avec une couverture forestière plus ou moins dense par l'endroit. La classe culturejachère qui englobe de nombreuses cultures abandonnées et les plantations telles que le palmier et le cacaoyer se confondent aux forêts dégradées. Il faut souligner que dans la zone d'étude, les cultures pérennes telles que des plantations de cacaoyers sont généralement cultivées sous couvert forestier, ce qui rend souvent difficile la discrimination des formations (forêt dense, forêt dégradée, plantation, cultures et jachères). Cette même remarque avait été faite par N'Guessan et al., (2006) dans l'étude sur le suivi par télédétection spatiale d'une forêt tropicale humide protégée soumise à des pressions anthropiques au niveau de la forêt classée du Haut-Sassandra en Côte d'Ivoire. Les classes d'occupations du sol identifiées par N'DA (2007) et Akadje et al., (2014), ont des précisions supérieures de 50\% et avec une part plus importante ayant des précisions supérieures de $90 \%$. On note ainsi que nos résultats sont plus précis que ceux de N'DA et d'Akadje et al pour l'image de 1986.

\section{Dynamique de l'occupation du sol dans les forêts de l'axe Impfondo- Dongou}

La dynamique des changements a fait ressortir les différents processus d'évolution intervenus au sein du paysage entre 1986-2015. Une augmentation des surfaces des forêts dégradées et des cultures et jachères au dépend des autres catégories tels que la forêt primaire a été observée. Cette perte serrait due aux pratiques agricoles, à la production du bois de chauffe et à l'exploitation artisanale, car la zone d'étude constitue en fait une zone où les cultures pérennes comme le palmier à huile et le cacao prédominent. C'est également une zone où les cultures vivrières telles que le manioc dominent d'où la dégradation avancée de la forêt primaire au profit de l'agriculture. La réduction des forêts peut aussi s'expliquer par le type 
d'agriculture pratiquée par les populations. En effet, après deux ou cinq années de cultures vivrières, les paysans abandonnent les parcelles en cultures pour coloniser de nouvelles terres forestières plus fertiles, laissant les premières en jachère afin de restaurer leur fertilité. Cette pratique agricole est associée à des feux de végétation et à l'abattage des arbres pour la fabrication de pirogues, des constructions des maisons, la récolte du miel et l'obtention du bois de chauffe. Ces différentes activités ont par ailleurs un impact sérieux sur la biodiversité végétale des forêts de la zone d'étude (Ifo et al. 2016).

Il faut aussi noter que ces dernières années, l'accroissement de la population a engendré une forte extension urbaine ainsi que la création de plusieurs villages ou campements. Ce qui a pour conséquence la pression sur les ressources forestières.

Dans une étude réalisée sur le changement du couvert forestier entre 2000 et 2010, GAF, (2013), a montré que dans la partie nord du Congo, la construction des nouvelles routes ainsi que l'expansion des colonies existantes, les activités d'abattage sélectif et les plantations de palmiers à l'huile sont à l'origine de la déforestation et la dégradation des forêts.

Les résultats de l'étude réalisée sur l'occupation du sol au Togo en 2000, Kpogo et al., (2005), ont trouvé que la pression démographique, les anomalies climatiques, les feux plus fréquents, l'extension des cultures constituaient les causes de la dégradation des forêts togolaises. Ce constat a été fait Kokou et al., (2006) dans une étude sur la diversité des forêts denses du Togo. Ils ont montré que les causes de la dégradation des forêts sont d'origine anthropique.

Les résultats obtenus montrent que le changement du couvert forestier dans la zone d'étude est énorme. En effet, le taux de dégradation de 2001 à 2015 (8,25\% sur 14 ans, soit 0,59\% par an) était supérieur au taux de perte dans le département de la Likouala pour la période 2000-2010, ce taux a été estimé à $0,49 \%$ par FACET (2012) et à 0,32 \% par le GAF (2013). Par ailleurs, pour la période $1986-2015$, ce taux est chiffré à $0,43 \%$ et est supérieur au taux de perte national estimé à $0,062 \%$ pour la période 2000 2012 (CNIAF, 2015), mais inférieur au taux de perte national estimé à 0,75\%, pour la période 2000-2010 par le FACET (2012).

\section{Analyse de quelques paramètres explicatifs des changements de couverture forestière}

Plusieurs causes directes expliquent la perte des forêts dans le département de la Likouala parmi lesquelles : 


\section{La croissance urbaine}

La descente effectuée sur le terrain nous a révélé que la ville s'agrandit au fur et à mesure avec l'augmentation de la population. Par exemple pour la ville d'Impfondo, une étude a été réalisée sur la croissance urbaine révélant que cette communauté urbaine avait augmenté de 430,36 ha pendant la période 1986 à 2001 et de 320,01 ha pendant la période de 2001 à 2016 (Binsangou, 2016).

\section{Construction des infrastructures modernes}

La forte concentration des cultures et des sols anthropisés à proximité des routes montre que ces dernières sont bien un facteur d'appropriation de l'espace par les populations. La création de ces routes entraîne alors dans un premier temps des coupes importantes des forêts pour leur implantation, puis elles sont le point de départ de défrichements pour la mise en culture de nouveaux espaces (Leroux, 2012). D’après les résultats de GAF (2013), la déforestation due aux infrastructures est de $27,8 \%$ alors que la municipalisation a eu lieu dans le département en 2005.

\section{L’agriculture itinérante sur brulis}

L'agriculture est plus axée sur les cultures vivrières qui sont pour la plupart destinées à l'autoconsommation. Les cultures de rente pratiquées (cacao, café et palmier à huile) procurent l'essentiel des revenus aux paysans. Au fur et à mesure que la population augmente, la demande en produit agricole augmente et la production agricole augmente aussi. Dans ce département, une grande partie des forêts a été détruite par les populations locales pour la mise en place des cultures variables telles que : des champs de culture de manioc, palmier à huile, mais, bananier et bien d'autres. Ces cultures répondent à une diversité des demandes des populations urbaines. Cette activité est souvent réalisée par les populations non seulement pour leur alimentation, mais aussi pour l'obtention du revenu leur servant de satisfaire leurs besoins.

\section{L'exploitation forestière}

Partant du constat général d'une régression des formations végétales denses au profit des forêts dégradées, il convient d'analyser ces dynamiques au regard facteurs que sont notamment l'exploitation forestière artisanale, la récolte de bois de chauffe. Le département de la Likouala regorge plusieurs sociétés forestières d'exploitation. Cette opération qui permet d'extraire le bois, occasionne des pertes énormes de forêts. Le bois est toujours la principale source d'énergie domestique au Congo. Par conséquent, la demande de bois énergie (bois de chauffe) est entrain d'augmenter rapidement dans et autour des grands centre urbain.Les pirogues, pagaies, 
mortiers, et pétrins sont confectionnés chacun à l'aide des troncs d'arbres des essences spécifiques extraits de la forêt ( Entandophragma cylindricum, Milicia excelsa, Strombosia grandifolia Staudtia gabonensis ).

A ces causes ci-dessus citées, s'ajoutes les causes indirectes. Nous pouvons citer :

\section{Pression démographique}

Dans la zone d'étude, les surfaces emblavées sont en hausse au détriment des forêts. Lors de notre mission terrain, plusieurs raisons ont été évoquées pour justifier cette situation notamment l'augmentation de l'effectif de la population qui est passée de 154.115 habitants en 2007 à 196.877 habitants en 2015 avec une densité de 2,5 hbt/ $\mathrm{km}^{2}$ (RGPH 2007). Cet accroissement de la population oblige les paysans à produire plus pour les besoins d'alimentation. A cela s'ajoutent, la baisse de la fertilité des sols et donc des rendements des cultures vivrières. Ainsi, plus la population augmente, plus la pression sur les ressources disponibles augmente.

\section{L'arrivée des refugiés de la guerre civile}

Ayant fouis la guerre civile dans leurs pays respectifs : le Rwanda, la $\mathrm{RDC}$ et la Centrafrique, les populations de ces pays se sont installées dans le département de la Likouala. Les données collectées lors de notre visite sur le terrain auprès du service gouvernemental qui s'occupe de la gestion des réfugiés (CNAR), nous ont rapporté que dans la période de 2000 à 2014, 43290 réfugiés avaient été enregistrés dans ce département. Ces derniers ont contribué de façon significative à la perte de la couverture forestière, non seulement pour les besoins de construction des sites pour les hébergés mais aussi pour satisfaire leurs besoins.

Ces résultats confirment plusieurs recherches déjà réalisé sur les causes du changement de la couverture forestière parmi ces recherche nous pouvons cités les travaux de Geist et Lambin, 2001 ; Zhang et Guindon, 2005 ; Duveiller et al., 2008 ; Tchatchou et al., 2015 qui ont identifié les causes immédiates et les causes sou jacentes.

\section{Conclusion}

Cette étude porte sur le changement de couverture forestière dans le département de la Likouala durant la période 1986 - 2015. Les résultats issus de la cartographie ont indiqué des taux de déforestation estimés respectivement à $0,28 \%$ entre 1986 et 2001 et à $0,59 \%$ entre 2001 et 2015 . Cette baisse des surfaces forestières s'est faite au profit des zones agricoles et des zones dégradées qui ne cessent de conquérir de nouveaux espaces forestiers. 
Dans l'ensemble, les pressions anthropiques sur les surfaces forestières sont croissantes au regard des résultats obtenus. Cette étude nous a permis de comprendre qu'il y a une réelle nécessité d'alerter les pouvoirs publics de s'impliquer dans la gestion durable des forêts de la Likouala en général et de celles de l'axe Impfondo Dongou en particulier.

\section{Remerciement}

Les auteurs de cette étude remercient le projet GéoForafri pour le financement qui a permis la réalisation de cette étude. Nous voulons aussi remercier l'Université Marien N'GOUABI à travers la Direction de la Recherche et de la Coopération mais aussi les autorités administratives du Département de la Likouala pour toutes les facilitations.

\section{References:}

1. Adjonou K., Ali N., Kokutse. D. A, Novignon S. K. \& Kouami K., 2010. Étude de la dynamique des peuplements naturels de Pterocarpus erinaceus Poir. (Fabaceae) surexploités au Togo. Bois et Forêts des Tropiques, 306: 45-56.

2. Akadje L. Hauhouot A. C., 2014. Analyse par télédétection environnementales de 2000 à 2013 dans la zone Ramsar de GrandBassam (Sud de la Côte d'Ivoire). Resige, vol. 1, $\mathrm{n}^{\circ}$ 001, 33-40

3. Bannari A. Morin D. Huete A.R. \&Bonn F., 1995. A review of vegetation indices. Remote Sensing Review, $\mathrm{n}^{\circ} 13,95-120$

4. Barima Y. S. S., 2009. Dynamique, fragmentation et diversité végétale des paysages forestiers en milieux de transition forêt-savane dans le département de Tanda (Côte d' Ivoire). Thèse de doctorat, Université Libre de Bruxelles, Belgique.

5. Binsangou Malonga S., 2016. Suivi par télédétection de la déforestation due par la croissance urbaine de deux localités de la république du Congo : communauté urbaine d'Impfondo et la ville de Ouesso pendant la période de 1986 à 2016. Mémoire en vue d'obtention du diplôme de Master. Université Marien N'Gouabi, Ecole Nationale d'Agronomie et de Foresterie, 105p.

6. Caloz R.\& Collet C., 2001. Précis de télédétection. Volume 3: traitements numériques d'images de télédétection (Universités francophones). Presses Polytechniques du Québec, Canada.

7. Congalton R. G., 1991. A review of assessing the accuracy of classifications of remotely sensed data. Remote Sensing of Environment, 37: 35-46.

8. Duveiller G., Defourny P., Desclée B. et Mayaux P., 2008. Deforestation in Central Africa - estimates at regional national and 
landscape levels by advanced processing of systematically distributed Landsat extracts. Remote Sens Environment, 112: 1969 - 1981.

9. Forêts d'Afrique Centrale Evaluées par Télédétection (FACET), 2012. Étendue et perte du couvert forestier en République du Congo de 2000 à 2010. Observatoire Satellital des Forêts d'Afrique Centrale (OSFAC). 110p. http://osfac.net/component/k2/itemlist/category/184facet.

10. GAF, 2013. SGDE Suivi Forestier Extension des Services REDD. S - Rapport des opérations de service pour la République du Congo phase 3. Doc n ${ }^{\circ}$ GSE-REDD-S6-RoC-Ph3, issue/Rev-n¹.0, 74pp.

11. Geist H.J. et Lambin E. F., 2001. III Land-Use and Land-Cover Change (LUCC) Project IV. International Human Dimensions Programme on Global Environmental Change (IHDP) V. International Geosphere-Biosphere Programme (IGBP), LUCC Report Series.

12. GIEC., 2006. Lignes directrices 2006 du GIEC pour les inventaires nationaux de gaz à effet de serre. Vol. 4: Agriculture, foresterie et autres affectations des terres. Hayama, Japon, IGES pour le GIEC (disponible aussi sur: http://www. ipccnggip.iges.or.jp/public/2006gl/ french/index.html), 85p.

13. Ifo S. A., Moutsambote J-M., Koubouana F., Yoka J., Ndzai S.F., Bouetou-Kadilamio L. N. O., Mampouya H., Jourdain C., Bocko Y., Mantota A. B., Mbemba M., Mouanga-Sokath D., Odende R., Mondzali L. R., Mampouya Wenina Y. E., Ouissika B. C., and Loumeto J. J., 2016. Tree Species Diversity, Richness, and Similarity in Intact and Degraded Forest in the Tropical Rainforest of the Congo Basin: Case of the Forest of Likouala in the Republic of Congo. International Journal of Forestry Research Volume 2016, 12 pages. http://dx.doi.org/10.1155/2016/7593681

14. Kokou K., Atato a., Bellefontaine R., Kokutse and., Caballe G., 2006. Diversité des forêts sèches du Togo. Revue d'Ecologie, vol.61, $\mathrm{n}^{\circ} 3$, pp. 225-246

15. Kpogo Y. D. et Kokou E., 2005. Cartographie de 1'Occupation et de l'Utilisation du Sol au Togo, Rapport du Projet West Africa LandUse and Land-Cover, Centre Regional AGRHYMET, Niamey, Niger, $27 \mathrm{p}$

16. Leroux L., 2012. Analyse diachronique de la dynamique paysagère sur le bassin supérieur de l'Ouémé (Bénin) à partir de l'imagerie Landsat et MODIS-Cas d'étude du communal de Djougou, Rapport de travail, Hydrosciences Montpelier, France, ANR ESCAPE. 62p.

17. Makaya H. A. J., 2015. Evaluation des proportions de changements des forêts en République du Congo. Mémoire de fin de formation 
pour l'obtention du diplôme de Master Recherche. Option Gestion Durable des forêts, Ecole Nationale Supérieure d'Agronomie et de Foresterie, Université Marien Ngouabi, Congo, 48p.

18. Mama A., 2013. Antropisation des paysages au Benin : dynamique, fragmentation et développement agricole. Thèse de doctorat, Université Libre de Bruxelles, Belgique 216 pp.429-441.

19. Mamadou A. S., 2009. Cartographie des changements de l'occupation du sol entre 1990 et

20. 2002 dans le nord du Sénégal (Ferlo) à partir des images Landsat. Cybergeo: European Journal of Geography. URL: http://cybergeo.revues.org/index22707.html.

21. N'DA D. H., (2007). Etude et suivi par télédétection et système d'informations géographiques d'une aire protégée soumise aux pressions anthropiques : cas du Parc National de la Marahoué.Thèse de Doctorat 181 p. Université de Coccody, Côte d'Ivoire

22. Niveau des Emissions de Références pour les Forêts(NERF) en République du Congo, 2016.

23. N'Guessan E., Dibi N'Dah H., Bellan M-F. \& Blasco F., 2006. Pression anthropique sur une réserve forestière en Côte d'Ivoire : Apport de la télédétection. Télédétection, 5: 307-323.

24. Niveau des Emissions de Références pour les Forêts(NERF) en République du Congo, 2016.

25. Nkokolo Nkaya D., 2012. Suivi de la dynamique des jeunes tiges des essences de valeur commerciale dans les parcelles après exploitation: cas de l'AAC3-2012 de l'UFP1, l'UFA Mokabi-Dzanga, Société Mokabi Département de la Likouala. UMNG, IDR, BZV Congo 61P

26. Pham T. T. H., Bonn F. \& Dubois J-M., 2007. Démarche méthodologique pour la détection des changements d'un milieu morcelé en utilisant des images à moyenne résolution spatiale : application à une région littorale au Viêt-Nam. Télédétection, 1: 1-4.

27. Pontus R. G. J., 2000. Quantification error versus location in comparison of categorical maps. Photogrammetric Engineering and Remote Sensing, 66: 1011-1016.

28. Robin M., 2002. Télédétection, des Satellites au SIG. Une analyse complète du processus de création d'un type essentiel d'information géographique, 2ème édition, Nathan Université.

29. Shalaby A. \& Tateishi R., 2007. Remote sensing and GIS for mapping and monitoring land cover and land-use changes in the Northwestern coastal zone of Egypt. Applied Geography, 27: 28-41.

30. Skupinski G., Don B. T. \& Weber C., 2009. Les images satellites Spot multi-dates et lamétrique spatiale dans l'étude du changement urbain et suburbain. Le cas de la basse vallée de Bruche (Bas-Rhin) 
European Journal of Geography. URL : http://cybergeo.revues.org/21995; DOI :10.4000/cybergeo.21995. France

31. Souza C. M. Jr., Roberts D. A. and Cochrane M. A., 2005. Combining spectral and spatial information to map canopy damage from selective logging and forest fires. Remote Sensing of Environment 98(2-3):329-343.

32. Tchatchou B., Sonwa D.J., IFO S. et Tiani A. M., 2015. Déforestation et dégradation des forêts dans le bassin du Congo : Etat des lieux, causes actuelles et perspectives. Papier occasionnel 120. Bogor, Indonésie : CIFOR, 47p.

33. Yangambi, 1956. C.S.A. Specialist Meeting on Phytogeography / Réunion de spécialistes du C.S.A. en matière de phytogéographie. Scientific Council for Africa South of the Sahara, Yangambi, C.C.T.A, 35 p.

34. Vennetier P., 1966. - Atlas du Congo. J.A., 38p.

35. Zhang Y. et Guindon B., 2005. Landscape Assessment of Human Impacts on Deforestation in the Great Lakes Watershed; Canada Journal of Remote Sensing, vol. 31, pp: $153-166$. 